\title{
Introdução de alimentos industrializados e de alimentos de uso tradicional na dieta de crianças de creches públicas no município de São Paulo ${ }^{1}$
}

\section{$\int\left(\int_{\substack{0+0 \\ \text { date }}}\right.$}

Introduction of processed and traditional foods

to the diets of children attending public

daycare centers in São Paulo, Brazil

Maysa Helena de Aguiar TOLONI²

Giovana LONGO-SILVA²

Rita Maria Monteiro GOULART ${ }^{3}$

José Augusto de Aguiar Carrazedo TADDEI ${ }^{2}$

RE S U M O

\section{Objetivo}

Descrever e discutir a introdução de alimentos industrializados na dieta de crianças frequentadoras de berçários em creches, considerando a recomendação do Ministério da Saúde para uma alimentação saudável.

\section{Métodos}

Estudo transversal com 270 crianças frequentadoras de berçários de 8 creches públicas e filantrópicas do município de São Paulo. Por meio de questionário estruturado e pré-codificado, foi avaliada a introdução de alimentos a partir de 11 perguntas. Para cada alimento analisado foi registrada a idade em meses de introdução e avaliada a concordância com o oitavo passo do Guia Alimentar. No estudo das associações, utilizou-se o teste Qui-quadrado, a partir das variáveis idade e escolaridade maternas, renda familiar e trabalho da mãe fora do lar.

\section{Resultados}

Os resultados mostram que para aproximadamente 2/3 das crianças foram oferecidos, antes dos 12 meses, alimentos com potencial obesogênico, como macarrão instantâneo, salgadinhos, bolacha recheada, suco artificial, refrigerante e bala/pirulito/chocolate. São os filhos de mães com baixa escolaridade, mais jovens e com menor renda, os mais susceptíveis ao erro alimentar de introdução precoce de alimentos industrializados.

\footnotetext{
1 Apoio: Fundação de Amparo à Pesquisa do Estado de São Paulo (Processo nº 2006/02597-0).

2 Universidade Federal de São Paulo, Departamento de Pediatria. R. Loefgreen, 1647, Vila Clementino, 04040-032, São Paulo, SP, Brasil. Correspondência para/Correspondence to: J.A.A.C. TADDEI.E-mails: <taddei.dped@epm.br>; <nutsec@yahoo.com.br>.

${ }^{3}$ Universidade São Judas Tadeu, Departamento de Graduação em Nutrição. Butantã, SP, Brasil.
} 
62 | M.H.A. TOLONI et al.

\section{Conclusão}

Diante desses resultados, medidas educativas e preventivas devem ser propostas para a formação de hábitos alimentares saudáveis desde a infância, além da criação de campanhas abrangentes e efetivas que estimulem o consumo de frutas e hortaliças, considerando-se os fatores culturais, comportamentais e afetivos envolvidos com a alimentação.

Termos de indexação: Alimentos industrializados. Comportamento alimentar. Consumo de alimentos. Hábitos alimentares. Nutrição do lactante.

\section{A B S T R A C T}

\section{Objective}

This study described and discussed the introduction of processed foods to the diets of children attending the nurseries of daycare centers, considering the recommendation of the Ministry of Health for a healthy diet.

\section{Methods}

This cross-sectional study included 270 children attending nurseries of eight public and not-for-profit daycare centers in São Paulo city. A pre-coded and structured 11-question questionnaire was used to evaluate the introduction of processed foods. For each type of food analyzed, the corresponding age in months was recorded as well as assessed as to whether it was in agreement with the 8th step of the Dietary Guide. The chi-square test was used for determining the associations. The studied variables were mother's age, education level and working status and family income.

\section{Results}

The results showed that approximately 2/3 of the studied children under 12 months of age were offered foods with obesogenic potential, such as instant noodles, snacks, sandwich cookies, powdered juice, soft drinks and candy/lollipop/chocolate bars. Children born to younger mothers, with low education level and lower income are most vulnerable to the feeding error of introducing processed foods prematurely.

\section{Conclusion}

These results show that educational and preventive actions should be proposed to build healthy eating habits from childhood. Efficient and in-depth campaigns aiming at promoting the consumption of fruits and vegetables are also needed, taking into consideration the cultural, behavioral and emotional factors associated with diet.

Indexing terms: Industrialized foods. Feeding behavior. Food consumption. Eating habits. Infant nutrition.

\section{N T R O D U ÇÃ O}

A alimentação está intimamente associada à saúde, nutrição, crescimento e desenvolvimento infantil, constituindo-se os primeiros anos de vida em período vital para o estabelecimento de práticas alimentares adequadas, que são, por sua vez, condicionadas pelo poder aquisitivo e nível de informação das famílias ${ }^{1-4}$.

Nos últimos anos, grandes mudanças ocorreram nos hábitos alimentares da população, principalmente em relação à substituição de alimentos caseiros e naturais por alimentos industrializados, sendo estes responsáveis por $85 \%$ do consumo total de alimentos em 20075,6. Vários fatores contribuíram para tais mudanças, entre eles a influência do mercado publicitário, a globalização, o ritmo acelerado de vida nas grandes cidades e o trabalho da mulher fora do lar. O desenvolvimento econômico e social ampliou consideravelmente o acesso efetivo aos alimentos prontos para o consumo, favorecendo maior participação dos extratos sociais de menor renda ${ }^{4,7,8}$.

A introdução de alimentos altamente energéticos e de baixo valor nutricional desde o início da vida, bem como o abandono precoce do aleitamento materno, contribuem para o comprometimento do crescimento e desenvolvimento da criança, além de propiciar a diminuição da proteção imunológica e o desencadeamento de processos alérgicos e distúrbios nutricionais ${ }^{3,9}$. Dessa forma, alimentação hiperenergética, com consu- 
mo excessivo de açúcares simples, gorduras animais, ácidos graxos saturados, gordura trans, sódio e redução dos carboidratos complexos e fibras, associados ao sedentarismo, são fatores responsáveis pelo surgimento precoce das Doenças Crônicas Não-Transmissíveis (DCNT)7,10,11.

Diante disso, o Ministério da Saúde (MS) elaborou em 2002, juntamente com a Organização Pan-Americana da Saúde (OPAS) recomendações expressas em "Dez passos para uma alimentação saudável: guia alimentar para crianças menores de dois anos", publicadas em um manual técnico para subsidiar os profissionais de saúde a promover práticas alimentares saudáveis. O oitavo passo desse Guia Alimentar recomenda evitar açúcar, café, enlatados, frituras, refrigerantes, balas, salgadinhos e outras guloseimas nos primeiros anos de vida e usar sal com moderação. Substâncias presentes nesses alimentos podem irritar a mucosa gástrica da criança, que possui maior sensibilidade no primeiro ano de vida, podendo comprometer a digestão e a absorção de nutrientes ${ }^{12-14}$. Além disso, elas contêm aditivos e conservantes artificiais que, embora dentro dos limites máximos preconizados pela legislação, ainda não foram devidamente testados para uso a longo prazo, desde a infância até a idade adulta ${ }^{15}$.

Diante do exposto e tendo em vista as potenciais consequências deletérias de uma alimentação inadequada na infância, este trabalho teve como objetivo descrever e discutir a introdução de alimentos industrializados e de uso tradicional na dieta de crianças frequentadoras de berçários em creches, considerando a recomendação do Ministério da Saúde para uma Alimentação Saudável.

\section{M É T O D O S}

Trata-se de estudo transversal, sendo parte integrante da primeira fase de avaliação pré-intervenção de um Projeto mais amplo, do tipo ensaio institucional randomizado com controle concomitante e tempo de acompanhamento de um ano. O "Projeto Crecheficiente" - Impacto do treinamento de educadoras de creches públicas/filantrópicas nas práticas higiênico-dietéticas e na saúde/nutrição dos lactentes - compreende um estudo composto por duas fases e que tem como objetivo avaliar o efeito do treinamento de educadoras de creche na saúde dos lactentes atendidos e na aquisição de conhecimentos e mudanças nas atividades desenvolvidas.

No início do Projeto, foram contatadas 36 creches subordinadas à Coordenadoria da Educação de Santo Amaro, na cidade de São Paulo, tendo sido 16 delas visitadas pela equipe do Projeto. Após o contato inicial e visitas para coleta de informações, aplicou-se a metodologia de seleção proposta por Beghin et al. ${ }^{16}$, considerando, como critérios de prioridade, maior número de educadoras de berçário, maior número de lactentes, segurança para execução da pesquisa, ausência de intervenção prévia de educação em saúde, receptividade e facilidade de acesso à instituição. Foram então selecionadas 4 creches públicas e 4 filantrópicas.

A população de estudo foi composta por 270 crianças, de ambos os sexos, com faixa etária entre 4 e 29 meses, que frequentavam regularmente os berçários de 8 creches públicas e filantrópicas do município de São Paulo e que foram autorizadas pelos pais ou responsáveis a participar da pesquisa, mediante assinatura do termo de consentimento informado, livre e esclarecido. Esse número de crianças é suficiente para identificar razões de risco da ordem de 1,17 (alfa=0,05, beta $=0,2)$. O tamanho da amostra foi mensurado no Programa Epi Info 2000, versão 3.4.317.

Foram excluídas as crianças portadoras de síndrome de Down $(n=2)$, paralisia cerebral $(n=2)$, síndrome genética $(n=1)$, aquelas cujos pais ou responsáveis recusaram-se a assinar o termo de consentimento $(n=2)$ e as que não estavam presentes no dia da coleta de dados $(n=3)$, totalizando uma perda amostral de 3,6\%.

A coleta de dados foi efetuada pela equipe de pesquisadores do Projeto, compreendida por cinco nutricionistas, um pediatra e um dentista devidamente treinados, que realizaram entrevistas 
$64 \mid$ M.H.A. TOLONI et al.

com os pais ou responsáveis pelas crianças, no período de maio a junho de 2007.

Um questionário estruturado e pré-codificado, composto por perguntas abertas e fechadas, foi elaborado e previamente testado para a coleta de dados. As variáveis estudadas foram: idade e escolaridade maternas, renda familiar, trabalho da mãe fora do lar e dados sobre alimentação infantil. Essas variáveis foram dicotomizadas, baseadas em pontos de corte encontrados em outras pesquisas, nos valores reconhecidos e recomendados por órgãos oficiais ou no valor das medianas da amostra estudada ${ }^{12,18}$.

Para o preenchimento desse instrumento, foi elaborado um manual com orientações aos entrevistadores e com a codificação das variáveis, a fim de uniformizar a anotação e a análise dos dados coletados. Todos os questionários foram avaliados quanto a sua consistência interna antes de serem digitados. Os dados foram transcritos em banco de dados com dupla digitação e posterior validação, visando à correção de erros.

A introdução de alimentos foi avaliada a partir de 14 itens coletados por meio do ques- tionário aplicado. Para cada um dos alimentos analisados, foi registrada a idade em meses de introdução e avaliada a concordância com o oitavo passo do Guia Alimentar ${ }^{12}$.

Os dados obtidos foram analisados no programa estatístico Epi Info 2000, versão 3.4.317. Foram realizadas análises de consistência e estatísticas descritivas univariadas e bivariadas. No estudo das associações, utilizou-se o teste Qui-quadrado. O nível de significância adotado foi de $5 \%(p<0,05)$.

O projeto foi aprovado pelo Comitê de Ética e Pesquisa da Universidade ao qual está vinculado (processo 0273/08).

\section{RES ULTADOS}

Do total de crianças estudadas (270), observou-se pequena predominância do sexo masculino (53\%). A mediana de idade foi de 19 meses (amplitude de 4-29). Observou-se maior proporção de mães na faixa etária entre 20 e 35 anos de idade, com idade média de 27,5 anos.

Tabela 1. Distribuição percentual de crianças segundo introdução de alimentos industrializados e de uso tradicional, por faixa etária. São Paulo (SP), 2007.

\begin{tabular}{|c|c|c|c|c|c|c|c|c|}
\hline \multirow{3}{*}{ Alimentos } & \multicolumn{8}{|c|}{ Idade de introdução } \\
\hline & $0-3 m$ & $4-6 m$ & $7-9 m$ & $10-12 m$ & $>12 \mathrm{~m}$ & Não introduziu & Não informado & Total \\
\hline & $\%$ & $\%{ }^{*}$ & $\%^{*}$ & $\%{ }^{*}$ & $\%^{*}$ & $\%$ & $\%$ & $\mathrm{n}$ \\
\hline \multicolumn{9}{|l|}{ Industrializados } \\
\hline Gelatina & 5,2 & 42,2 & 61,8 & 88,1 & 90,3 & 9,7 & 0 & 270 \\
\hline Bala/pirulito/chocolate & 1,9 & 13,8 & 32,0 & 74,2 & 84,8 & 15,6 & 0 & 270 \\
\hline Macarrão instantâneo & 2,2 & 23,7 & 43,7 & 77,8 & 83,0 & 16,6 & 0,4 & 270 \\
\hline Salgadinhos & 1,5 & 13,4 & 28,2 & 73,7 & 80,7 & 19,3 & 0 & 270 \\
\hline Bolacha recheada & 1,1 & 16,3 & 35,2 & 71,1 & 79,2 & 20,8 & 0 & 270 \\
\hline Suco artificial & 2,2 & 18,1 & 32,5 & 63,6 & 71,7 & 27,9 & 0,4 & 270 \\
\hline Embutidos & 0,4 & 10,1 & 18,2 & 58,2 & 67,1 & 32,9 & 0 & 270 \\
\hline Refrigerante & 1,1 & 12,2 & 19,6 & 56,5 & 66,7 & 32,9 & 0,4 & 270 \\
\hline \multicolumn{9}{|c|}{ Alimentos de uso tradicional } \\
\hline Açúcar & 31,1 & 63,3 & 71,8 & 87,0 & 90,0 & 10,0 & 0 & 270 \\
\hline Chá & 49,3 & 81,5 & 85,9 & 88,1 & 88,1 & 10,8 & 1,1 & 270 \\
\hline Mel & 18,2 & 51,5 & 60,4 & 73,7 & 76,7 & 22,2 & 1,1 & 270 \\
\hline Espessantes & 12,6 & 47,8 & 63,0 & 69,6 & 70,7 & 28,9 & 0,4 & 270 \\
\hline Fritura de imersão & 0 & 5,6 & 13,0 & 52,3 & 61,6 & 38,0 & 0,4 & 270 \\
\hline Café & 0,4 & 6,7 & 12,3 & 30,8 & 40,8 & 58,8 & 0,4 & 270 \\
\hline
\end{tabular}

*Percentual acumulado. 
Tabela 2. Distribuição de crianças segundo introdução de alimentos industrializados, prevalências e Odds Ratios, com seus respectivos intervalos de confiança (IC 95\%) e variáveis maternas. São Paulo (SP), 2007.

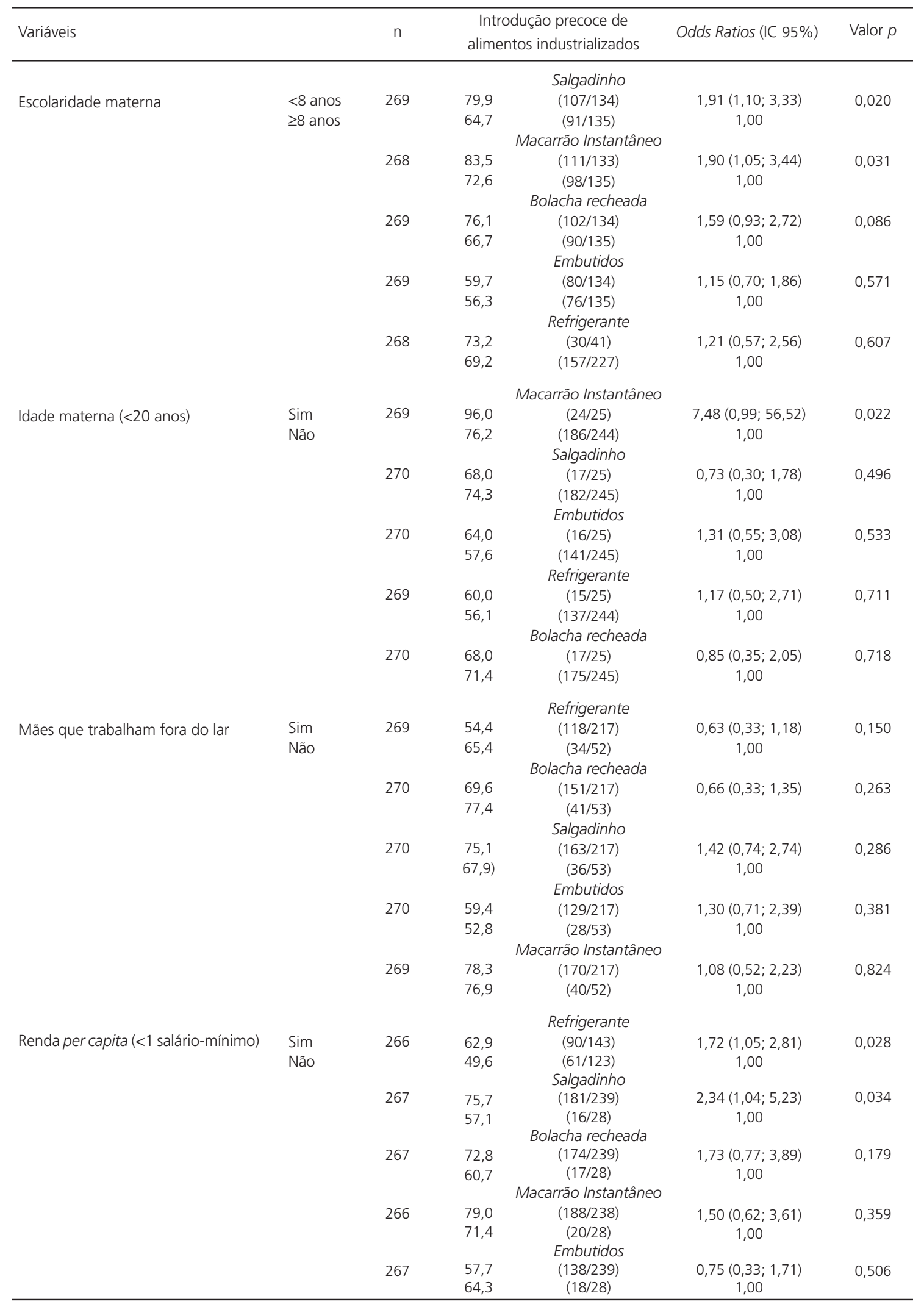


66 | M.H.A. TOLONI et al.

Em relação à escolaridade materna, verificou-se que $49,8 \%$ das mães tinham menos de 8 anos de estudo.

A Tabela 1 apresenta a frequência acumulada (\%) das crianças estudadas, segundo a faixa etária de introdução dos alimentos industrializados e de consumo tradicional. Observa-se que para aproximadamente $2 / 3$ das crianças foram oferecidos, antes dos 12 meses, alimentos com potencial obesogênico, como macarrão instantâneo, salgadinhos, bolacha recheada, embutidos, suco artificial, refrigerante e bala/pirulito/ chocolate.

Na Tabela 2, são apresentadas as associações entre a introdução, até os 12 meses de idade, dos cinco alimentos industrializados mais citados pelas mães na dieta das crianças frequentadoras dos berçários e os principais fatores de risco relacionados. As frequências destes e as análises individuais de seus efeitos apresentam-se ordenados por nível de significância estatística. Evidencia-se que baixa escolaridade materna duplica o risco de introdução precoce de salgadinho e macarrão instantâneo, enquanto que idade materna inferior a 20 anos corresponde a riscos aproximadamente 7,5 vezes maiores de introdução precoce do macarrão instantâneo. A renda per capita familiar inferior a um salário-mínimo representa também riscos cerca de duas vezes maiores para a introdução precoce de refrigerante e salgadinhos.

Conclui-se na análise da Tabela 2 que, mesmo na população de baixa renda estudada, são os filhos de mães com baixa escolaridade, mais jovens e com menor renda, os que estão mais susceptíveis ao erro alimentar de introdução precoce dos alimentos industrializados.

\section{DISCUSSÃO}

Apesar da inexistência de recomendações específicas quanto à quantidade e frequência do consumo de alimentos industrializados na dieta infantil, sabe-se que eles devem ser desencora- jados nos primeiros anos de vida, devido às fortes evidências de que sua ingestão continuada promove o desenvolvimento precoce das DCNT. Segundo alguns estudos, esse consumo pode ser influenciado por alguns fatores como renda familiar, idade e escolaridade maternas 2,9,14.

Os dados aqui apresentados refletem a realidade das creches públicas e filantrópicas do município de São Paulo, uma vez que, embora não se tenha feito uma amostragem probabilística, buscou-se incluir creches que refletissem o universo dessas instituições. Logicamente o estudo de natureza transversal está sujeito ao viés recordatório, podendo não ser precisas as informações das mães ou responsáveis. Estudo longitudinal, embora desejável, pressuporia a necessidade de tempo e recursos financeiros não disponibilizados para a equipe de pesquisa.

A análise da introdução de alimentos na dieta das crianças estudadas revelou que foram oferecidos, antes dos 12 meses de vida, chá para 88\% e açúcar para 87\% delas, os quais são alimentos de uso tradicional, porém não recomendados para essa faixa etária. A principal justificativa para a prática deve-se ao fator cultural que, na concepção das mães e avós, associa o chá a propriedades calmantes, ao alívio de cólicas e à hidratação. Esse erro alimentar resulta em consumo de menor quantidade de leite materno, interferindo ainda na biodisponibilidade de micronutrientes importantes, como o ferro e o zin$\mathrm{CO}^{2,3,9,19}$.

O uso precoce de açúcar, principalmente no chá e no leite não-materno, também faz parte de hábitos culturalmente estabelecidos e da tendência das mães de oferecerem alimentos doces, para, na sua concepção, satisfazer o paladar da criança e deixá-la bem alimentada. Esse alimento contribui para o aumento da densidade energética, possui índice glicêmico elevado e é isento de outros nutrientes importantes para a criança. Seu consumo leva ao aumento do conteúdo energético da dieta, sem melhorar o valor nutritivo da preparação à qual é acrescido, levando ainda ao aumento dos índices cariogênicos ${ }^{4,20,21}$. 
Com relação aos espessantes, já estavam introduzidos para $69,6 \%$ das crianças no primeiro ano de vida. Esse dado remete a traços culturais, já que popularmente se acredita que seu uso seja uma opção saudável e tradicional para saciar e alimentar a criança, em razão de sua predominante composição por carboidratos simples, que contribuem para $90 \%$ a $100 \%$ da densidade energética do produto. Anteriormente aos processos de industrialização e globalização, quando as carências nutricionais eram prevalentes, os espessantes já não eram recomendados. No cenário atual da transição nutricional, as principais preocupações dietéticas se voltam aos excessos alimentares, e a utilização inadequada dos espessantes constitui erro alimentar a ser corrigido com medidas de educação nutricional9,22.

Quanto ao mel, para 73,7\% das crianças estudadas havia sido oferecido antes dos 12 meses de idade. É um dado preocupante, considerando que a Agência Nacional de Vigilância Sanitária (Anvisa) recomenda que esse alimento não seja consumido no primeiro ano de vida, devido à imaturidade da flora intestinal, que torna as crianças mais susceptíveis à intoxicação alimentar, causada pela ingestão de esporos da bactéria Clostridium botulinum, bacilo responsável pela transmissão do botulismo intestinal23,24.

A introdução de refrigerantes e sucos artificiais ocorreu até os 12 meses de idade em $56,5 \%$ e $63,6 \%$ das crianças, respectivamente. Além de conterem aditivos químicos, esses produtos têm sido alvo de intensa preocupação, pois apresentam elevado teor de açúcar e, na sua maioria, são desprovidos de micronutrientes. Mais grave ainda, a sua introdução precoce vem substituindo os sucos de frutas naturais, ricos em vitaminas e minerais, e que contribuem significativamente para a ingestão diária recomendada nesse estágio da vida, principalmente a vitamina $C$, que potencializa o aproveitamento do ferro dietético 25,26 .

Vale ressaltar ainda que os refrigerantes contêm polifenóis que interferem na absorção de ferro não-heme, podendo contribuir para a ocor- rência da anemia nessa faixa etária ${ }^{27,28}$. Têm também teores excessivos de acidulante como ácido fosfórico, cujo consumo está associado ao aparecimento precoce de osteoporose ${ }^{14,29}$.

A Tabela 2 mostra a associação entre as variáveis preditoras da introdução de alimentos industrializados na dieta infantil e os cinco alimentos emblemáticos mais precocemente utilizados nessa faixa etária. Houve associação estatisticamente significante entre a introdução precoce dos refrigerantes e dos salgadinhos e a baixa renda familiar. Outros estudos sugerem que, tanto em países desenvolvidos como em desenvolvimento, o consumo de alimentos industrializados de baixo valor nutritivo está associado ao menor nível socioeconômico da população 4,30.

Assim, o risco de introdução precoce dos refrigerantes e dos salgadinhos é cerca de duas vezes maior entre famílias com renda inferior a um salário mínimo, quando comparadas com os lactentes de famílias que, embora pertencentes aos extratos de baixa renda, já que têm seus filhos matriculados em creches públicas e filantrópicas, têm renda superior a um salário mínimo per capita. Tal relação seria provavelmente mais forte caso fossem incluídos extratos de melhor condição socioeconômica.

Além disso, há grande influência da propaganda, veiculada principalmente pela televisão, visto que esses produtos são alvo de intensas campanhas publicitárias, além do forte investimento promocional que recebem nos pontos de comercialização ${ }^{11,31}$. Alguns estudos citam a influência da publicidade sobre a confiança que as mães depositam nos produtos apresentados nos comerciais, principalmente devido à falta de conhecimento e à desinformação ${ }^{19,32}$. Nesse contexto, Almeida et al. ${ }^{8}$, avaliando 1395 anúncios de produtos alimentícios veiculados na televisão, observaram que cerca de $60 \%$ deles estão no grupo da pirâmide alimentar representado por gorduras, óleos, açúcares e doces, além de não existir publicidade de frutas e hortaliças.

Apesar da existência de leis que autor-regulamentam as práticas publicitárias no Brasil, 
68 | M.H.A. TOLONI et al.

é necessário informar melhor a população sobre a importância da escolha de alimentos saudáveis e controlar as estratégias de marketing agressivas, promovidas pelas empresas comercializadoras de alimentos industrializados, que enganosamente aparecem nas propagandas associados à saúde, beleza, bem-estar, juventude, energia e prazer $^{8,33,34}$.

Neste estudo, a escolaridade materna associou-se de forma estatisticamente significante com a introdução precoce de macarrão instantâneo e salgadinhos. A baixa escolaridade materna associa-se com menor poder aquisitivo, falta de acesso a informações em saúde e consequente escolha incorreta de alimentos para a criança, além de maior susceptibilidade à influência da publicidade, como já descrito.

O macarrão instantâneo, precocemente oferecido para $77,8 \%$ das crianças até os 12 meses, vem apresentando forte tendência de aumento no consumo, principalmente entre a população infantil, por ser um alimento de fácil aceitação e de preparo rápido e prático. Contudo, uma embalagem comercial ultrapassa $459 \%$ da recomendação diária de sódio e atinge $40 \%$ do nível de gordura total para essa faixa etária ${ }^{25,26}$. Esse alimento também apresentou associação de risco estatisticamente significante com a idade materna (<20 anos), fato esse que pode estar relacionado ao envolvimento das mães adolescentes em outras atividades, como estudo e/ou trabalho, prejudicando a introdução alimentar de forma gradativa. São consequências do estilo de vida da sociedade contemporânea, levando à integração precoce das crianças aos alimentos obesogênicos e aterogênicos erroneamente consumidos pelos adultos ${ }^{22}$. Por outro lado, as mães mais jovens têm também maiores possibilidades de ter sido influenciadas na infância e adolescência pelo processo de informação enganosa veiculada nas propagandas televisivas.

Geralmente consumidos entre as refeições e até mesmo substituindo-as, os salgadinhos e bolachas recheadas apresentam altos teores de gorduras, sal e açúcares, além de conservantes, corantes e outros aditivos alimentares. A ingestão continuada e excessiva pode definir e cronificar hábitos alimentares inadequados que perduram da adolescência até a idade adulta, contribuindo para a obesidade infantil e surgimento cada vez mais precoce das DCNT ${ }^{10,14}$.

Observou-se neste estudo que os alimentos industrializados são muito utilizados na alimentação infantil e que houve introdução precoce da maioria deles, por serem práticos e saborosos. No entanto, sua ingestão deveria ser controlada para evitar problemas de saúde à criança, e, segundo a recomendação do oitavo passo do Guia Alimentar do MS, os alimentos industrializados somente deveriam ser introduzidos após o primeiro ano de vida. Seu consumo geralmente está acompanhado por uma baixa ingestão de frutas, legumes, cereais e leguminosas ${ }^{35}$. Isso acontece talvez por desinformação das mães sobre a idade adequada para a introdução desses alimentos, assim como por falta de orientação e utilização do Guia Alimentar, instrumento de fácil acesso e compreensão para a população em geral, principalmente nos serviços básicos de saúde ${ }^{12}$.

Vale ressaltar que o ingresso dos lactentes na creche pode ser fator de risco para a introdução precoce dos alimentos, geralmente inadequados para essa faixa etária, bem como levar à interrupção precoce do aleitamento materno. As creches deveriam ser instituições que incentivam a formação dos hábitos alimentares saudáveis desde a infância, porém o que se observa na prática alimentar são cardápios monótonos e compostos por alimentos industrializados.

A implementação dos "Dez passos para uma alimentação saudável: guia alimentar para crianças menores de dois anos", segundo Vítolo et al. ${ }^{13}$, mostrou-se efetiva na melhora de alguns aspectos da saúde da criança (aleitamento materno, práticas alimentares e morbidades), sendo evidente a necessidade da ampla aplicação desse programa na rede básica de saúde e na comunidade. 


\section{CONCLUSÃO}

Nessa perspectiva, as consequências da introdução e da utilização precoce e incorreta de alimentos industrializados poderão ser amenizadas por iniciativas de educação nutricional, que atinjam parcela significativa da população. Além de conscientizar os pais sobre a idade adequada e a forma correta de introdução de alimentos, é necessário alertá-los sobre os malefícios causados pela introdução errônea e precoce dos industrializados, considerando os fatores culturais, comportamentais e afetivos envolvidos com a alimentação.

Faz-se necessário também a criação de campanhas abrangentes e efetivas que estimulem o consumo de frutas e hortaliças, associadas a medidas de restrição da propaganda de alimentos infantis.

Dessa forma, sugere-se que sejam realizados estudos que procurem medir a efetividade, a curto, médio e longo prazo, de diferentes intervenções de promoção nutricional que visem estimular a adoção de hábitos alimentares saudáveis nos primeiros anos de vida.

\section{COLABORADORES}

M.H.A. TOLONI responsável pela coleta, análise de dados e redação. G. LONGO-SILVA co-responsável pela coleta, análise de dados e redação. R.M.M. GOULART co-responsável pela idealização do projeto e revisão da redação. J.A.A.C. TADDEl responsável pela ideali-zação do projeto e revisão da redação.

\section{REFERÊ N CIAS}

1. Briefel RR, Reidy K, Karwe V, Devaney B. Feeding infants and toddlers study: Improvements needed in meeting infant feeding recommendations. J Am Diet Assoc. 2004; 104(1 Suppl 1):s31-s7.

2. Oliveira LPM, Assis AMO, Pinheiro SMC, Prado MS, Barreto ML. Alimentação complementar nos primeiros dois anos de vida. Rev Nutr. 2005; 18(4): 459-69. doi: 10.1590/S1415-527320050004000 02.
3. Barbosa MB, Palma D, Bataglin T, Taddei JAAC. Custo da alimentação no primeiro ano de vida. Rev Nutr. 2007; 20(1):55-62. doi: 10.1590/S1415-527 320070001000006.

4. Aquino RC, Philippi ST. Consumo infantil de alimentos industrializados e renda familiar na cidade de São Paulo. Rev Saúde Pública. 2002; 36(6):655-60.

5. Instituto Brasileiro de Geografia e Estatística. Pesquisa de Orçamentos Familiares no Brasil (POF) 2002-2003. Rio de Janeiro: IBGE; 2004 [citado 2008 nov. 17]. Disponível em: <http://www.ibge. gov.br>.

6. Associação Brasileira das Indústrias da Alimentação. Indústria da alimentação: balanço anual 2007 e perspectivas para 2008. São Paulo: ABIA; 2008.

7. Batista-Filho M, Souza Al, Miglioli TC, Santos MC. Anemia e obesidade: um paradoxo da transição nutricional brasileira. Cad Saúde Pública. 2008; 24(Suppl 2):S247-S57.

8. Almeida SS, Nascimento PCBD, Quaioti TCB. Quantidade e qualidade de produtos alimentícios anunciados na televisão brasileira. Rev Saúde Pública. 2002; 36(3):353-5.

9. Simon VGN. Introdução de alimentos complementares e sua relação com variáveis demográficas e socioeconômicas, em crianças no primeiro ano de vida, nascidas em Hospital Universitário no município de São Paulo. Rev Bras Epidemiol. 2003; 6(1): 29-38.

10. Jahns L, Siega-Riz AM, Popkin BM. The increasing prevalence of snacking among US children from 1977 to 1996. J Pediatr. 2001; 138(4):493-8.

11. Jackson P, Romo M, Castilio M, Castilio-Durán C. Las golosinas en la alimentación infantil: análisis antropológico nutricional. Rev Méd Chile. 2004; 132(10):1235-42.

12. Brasil. Ministério da Saúde. Dez passos para uma alimentação saudável: guia alimentar para crianças menores de 2 anos. Brasília: Ministério da Saúde; 2002.

13. Vítolo MR, Bortolini GS, Feldens CA, Dachler ML. Impacto da implementação dos dês passos de alimentação saudável para crianças: ensaio de campo randomizado. Cad Saúde Pública. 2005; 21(5):1448-57.

14. del Rea SI, Fajardo Z, Solano L, Páez MC, Sánchez A. Pátron de consumo de alimentos en niños de una comunidad urbana al norte de Valencia, Venezuela. Arch Latinoam Nutr. 2005; 55(3): 279-86.

15. Schumann SPA, Polônio MLT, Gonçalves ECBA. Avaliação do consumo de corantes artificiais por lactentes, pré-escolares e escolares. Ciênc Tecnol Aliment. 2008: 28(3):534-9. 
70 | M.H.A. TOLONI et al.

16. Beghin I, Cap M, Dujardin B. A guide to nutritional assessment. Geneva: WHO; 1988.

17. Dean AG, Arner TG, Sangam S, Sunki GG, Friedman $R$, Lantinga $M$, et al. Epi Info 2000: a database and statistics program for public health professionals for use on Windows 95, 98, NT, and 2000 computers. Atlanta (GA): Centers for Disease Control and Prevention; 2000.

18. World Health Organization. Infant and young child nutrition. Procedings of the $55^{\text {th }}$ World Health Assembly; 2002 April 16; Geneva. p.1-21. World Health Organization (WHA55/15).

19. Spinelli MGN, Goulart RMM, Santos ALP, Gumiero LDC, Farhud CC, Freitas EB, et al. Consumo alimentar de crianças de 6 a 18 meses em creches. Rev Nutr. 2003; 16(4):409-14. doi: 10.1590/S1415-5 2732003000400004.

20. Ramos-Gomez FJ, Weintraub JA, Gansky AS, Hoover $\mathrm{Cl}$, Featherstone JD. Bacterial, behavioral and environmental factors associated with early childhood caries. J Clin Pediatr Dent. 2002; 26(2): 165-73.

21. Simon VGN, Souza JMP, Souza SB. Aleitamento materno, alimentação complementar, sobrepeso e obesidade em pré-escolares. Rev Saúde Pública. 2009; 43(1):60-9.

22. Bueno MB, Souza JMB, Souza SB, Paz SMRS, Gimeno SGA, Siqueira AAF. Riscos associados ao processo de desmame entre crianças nascidas em Hospital Universitário de São Paulo, entre 1998 e 1999: estudo de coorte prospectivo do primeiro ano de vida. Cad Saúde Pública. 2003; 19(5): 1453-60.

23. Barsanti C, Taddei JAAC. Botulismo infantil: atualização. Rev Paul Pediatr. 1995; 13(2):48-52.

24. Dewey KG. Approaches for improving complementary feeding of infants and young children. Geneva: World Health Organization; 2000.

25. Pennington JAT, Douglass JS. Food values of portions commonly used. $18^{\text {th }}$ ed. Baltimore (MD): Lippincott Williams \& Wilkins; 2005.

26. Institute of Medicine. Dietary reference intakes: recommended intakes for individuals, vitamins, foods and nutrition board. Washington (DC): National Academy Press; 2002.

27. Konstantyner T, Taddei JAAC, Oliveira MN, Palma $D$, Colugnati $F A B$. Riscos isolados e agregados de anemia em crianças freqentadoras de berçários de creches. J Pediatr (Rio de Janeiro). 2009; 85(3):209-16.

28. Taddei JAAC, Troster EJ, Machado EHS, Brasil ALD. Condição nutricional e prevalência de anemia em crianças matriculadas em creches beneficentes. Rev Paul Pediatr. 2005; 23(1):21-6.

29. Mazariegos-Ramos E, Guerrero-Romero F, Rodríguez-Moráis M, Lazcano-Burciaga G, Paniaqua $R$, Amato $D$. Consumption of soft drinks with phosphoric acid as a risk factor for the development of hypocalcemia in children: a casecontrol study. J Pediatr. 1995; 126(6):940-2.

30. Kinsey JD. Food and families' socioeconomic status. J Nutr. 1994; 124(9 Suppl):1878S-85S.

31. Costa TF, Pontes TE, Brasil ALD, Ferreira ABR, Taddei JAAC. Transição nutricional e desenvolvimento de hábito de consumo alimentar na infância. In: Dutra-de-Oliveira JE, Marchini JS, organizadores. Ciências nutricionais: aprendendo a aprender. São Paulo: Sarvier; 2008. p.543-63.

32. Brasil. Ministério da Saúde. A saúde pública e a regulamentação da publicidade de alimentos. Brasília: Ministério da Saúde; 2006.

33. Araújo MFM, Rea MF, Pinheiro KA, Schmitz BAS. Avanços na norma brasileira de comercialização de alimentos para idade infantil. Rev Saúde Pública. 2006; 40(3):513-20.

34. Agência Nacional de Vigilância Sanitária. Consulta Pública n 71/06, de 10 de novembro de 2006. Brasília: Ministério da Saúde: 2006 [citado 2008 nov 20]. Disponível em: <http://www.anvisa.gov. br>.

35. Gonzalez W, Jonas SJ, Frongillo EA. Restricting snacks in U.S. Elementary Schools is associated with higher frequency of fruit and vegetable consumption. J Nutr. 2009; 139:(1)142-4.

Recebido em: 18/2/2009

Versão final reapresentada em: 17/8/2009 Aprovado em: 12/5/2010 